\title{
Does COVID-19 Provide a Clue for Thrombosis in ITP?
}

\author{
Jecko Thachil, MD, FRC Path ${ }^{1}$ \\ ${ }^{1}$ Department of Haematology, Manchester University Hospitals, \\ Manchester, United Kingdom
}

Semin Thromb Hemost 2021;47:463-466.

One of the significant clinical manifestations of COVID-19 is thrombosis in the different circulatory beds, most commonly within pulmonary vasculature. Pathogenesis of this complication has not yet been elucidated, though some autopsy reports have shown high prevalence of microthrombi in various organs, including lungs, kidney, and heart. ${ }^{1,2}$ One of these reports suggested the possibility of circulating megakaryocytes (MKs) as being contributory to this thrombotic feature. ${ }^{2}$ The concept of MKs as contributory to thrombosis and a related complication, fibrosis, was recently reviewed. ${ }^{3}$ Similarly, MKs may be hypothesized to play a role in the development of thrombosis in patients with immune thrombocytopenia (ITP).

Epidemiological studies were conducted in patients with ITP, and thrombosis was noted in those receiving thrombopoietin receptor agonists (TPO-RA). ${ }^{4}$ These revealed an increased risk of thrombosis even in the absence of any ITPspecific treatments. Possible reasons are still being investigated but include some well-known thrombotic risk factors like older age, splenectomy, and antiphospholipid syndrome. ${ }^{4}$ One of the culprits may be the circulating MKs similar to that found in COVID-19. This is especially true since there is increased MK activity in both ITP and its treatment with TPO-RA.,

Beside some forms of leukemias, the concept of circulating MKs in other nonneoplastic conditions is not new with the first observations made over six decades ago. ${ }^{7}$ Despite these early findings, the idea that the large platelet precursors being present in the circulation and even producing platelets in the pulmonary vascular bed has been a matter of debate. Even while some experts support a link between lung MKs and cardiorespiratory diseases, many opposing advocates argue that the finding of MKs in lung fields is a gravity phenomenon seen only in the postmortem period. ${ }^{8}$ Elegant work from Lefrançais et al has perhaps put an end to the debate and cemented the importance of lung MKs in platelet production, and it can confidently be said that at least a part of platelet pool can be generated within the lungs. ${ }^{9}$ In addition to the lungs, the placenta has also been shown to
Address for correspondence Jecko Thachil, MD, FRC Path, Department of Haematology, Manchester Royal Infirmary, Oxford road, Manchester, M13 9WL, United Kingdom (e-mail: jecko.thachil@mft.nhs.uk).

be a site of platelet production. ${ }^{10}$ The lungs being "nonfunctional" in the fetus, perfusion studies of placentae were undertaken by investigators who were able to isolate MKs with copious cytoplasm from this fetal respiratory organ. ${ }^{10}$ This study gives additional proof to the concept of circulating MKs and platelet production in sites other than the bone marrow, including the lungs. In this context, we have to wonder what may be the role of these circulating MKs.

Thrombocytosis is a common accompaniment of inflammatory states. It is also known to the platelet biologists that inflammatory cytokines like interleukins can stimulate MKs to produce platelets. This is indeed the case as has been demonstrated in patients with lung inflammation. ${ }^{11}$ Hansen and Pedersen collected blood from cubital veins and analyzed for MKs in 30 patients with bronchitis or bronchopneumonia and identified significantly higher circulating MKs in these patients. ${ }^{11}$ It is possible that the circulating MKs participate in the host-defense process by releasing chemokines and producing many young, active platelets at the inflammatory site (e.g., the lungs). Since platelets do not have the synthetic machinery to respond to the various infectious or inflammatory stimuli in a rapid manner (despite recent evidence of platelet $\mathrm{mRNA}^{12}$ ), delivery of a large number of well-equipped platelets in the vicinity of infection or inflammation is far more advantageous than a distant release of platelets.

\section{But Do MKs Contribute to Thrombosis?}

Autopsy reports of patients who succumbed to COVID-19 provide proof to this possibility, along with the demonstration of several naked MK nuclei in the lung tissue. ${ }^{13}$ An Italian study reported on the autopsy data from nearly 40 patients and identified an increased number of CD61 antibody-positive MKs in the lung capillaries of almost all patients. ${ }^{14}$ They however did not correlate the MK load with the microthrombi which were omnipresent in these lung samples. The MKthrombosis link was, however, shown in an older publication, published online January 22, 2021
Issue Theme Maintaining Hemostasis and Preventing Thrombosis in COVID-19 -Part II; Guest Editors: Emmanuel J. Favaloro, PhD, FFSc (RCPA) and Giuseppe Lippi, MD. (c) 2021. Thieme. All rights reserved. Thieme Medical Publishers, Inc., 333 Seventh Avenue, 18th Floor, New York, NY 10001, USA
DOI https://doi.org/ 10.1055/s-0040-1722306. ISSN 0094-6176. 
wherein a significant correlation has been identified between MK number and fibrin microthrombi in lung biopsies of 22 patients who died from extensive burns. ${ }^{15}$ Returning to COVID-19, Rapkiewicz and colleagues found MKs in the microvasculature of the heart, renal glomeruli, and lungs. The important finding in this study was the colocalization of MKs with platelet-fibrin microthrombi in the pulmonary circulation and even in the heart microvasculature. ${ }^{2}$

It has recently been suggested that TPO can trigger MKbiased hematopoietic stem cells to express von Willebrand factor (VWF), which is upregulated during times of stress such as inflammation. ${ }^{16}$ MKs also contain ultralarge high-molecular-weight VWF multimers not present in plasma, which may be released in the inflammatory setting. ${ }^{17}$ Elevated VWF levels are a well-established risk factor for thrombosis and have been postulated as one mechanism for COVID-19 thrombosis. ${ }^{18,19}$ In relation to ITP, VWF levels were noted to be elevated especially in older patients (as mentioned before, a risk factor for thrombosis) in a study of 20 patients with platelet count less than $100 \times 10^{9} / \mathrm{L} .{ }^{20}$ This rebalanced hemostasis (high VWF levels in those with lower platelet counts) would explain the reduced bleeding tendency commonly observed in ITP patients despite the very low platelet counts, in comparison to hypoproliferative thrombocytopenia where there are fewer MKs and therefore possibly no increased VWF release. This balance may however be tipped toward thrombosis in patients with ITP when they acquire additional risk factors (e.g., advanced age, splenectomy, inflammatory diseases which cause secondary ITP). In addition, TPO-RA treatment could also influence the MK-TPO axis by increasing VWF levels and predispose some of these patients to develop thrombosis. Almost three decades ago, a significant increase in VWF ristocetin cofactor levels was identified in patients with ITP, but no relationship to thrombosis was described. ${ }^{21}$ More recently, a study published in abstract form described elevated plasma VWF levels in approximately 50 patients with ITP while the ADAMTS-13 (a disintegrin and metalloproteinase with a thrombospondin type 1 motif, member 13) levels were lower in the same patients compared with control subjects. ${ }^{22}$ An increased ratio of VWF:ADAMTS13 was suggested by these authors as possibly contributory to the pathogenesis of thrombosis in ITP. ${ }^{22}$ Such a relative ADAMTS-13 deficiency was also demonstrated in a cohort of 50 hospitalized COVID-19 patients. The study demonstrated an inverse correlation between D-dimer and ADAMTS13 suggesting high possibility of thrombotic microangiopathy, where the VWF-platelet axis plays a pathogenic role. ${ }^{23}$

In conclusion, circulating MKs may play a role in triggering thrombosis in patients with conditions like COVID-19 and ITP (and its treatment with TPO-RA). This is possibly linked to release of VWF by MKs, which are increased in number in response to increased TPO levels in these disease states. Future, large studies in this interesting area are warranted.

Conflict of Interest

J. T. reports personal fees from AMGEN, personal fees from NOVARTIS, outside the submitted work.

\section{References}

1 Fox SE, Akmatbekov A, Harbert JL, Li G, Quincy Brown J, Vander Heide RS. Pulmonary and cardiac pathology in African American patients with COVID-19: an autopsy series from New Orleans. Lancet Respir Med 2020;8(07):681-686

2 Rapkiewicz AV, Mai X, Carsons SE, et al. Megakaryocytes and platelet-fibrin thrombi characterize multi-organ thrombosis at autopsy in COVID-19: a case series. EClinicalMedicine 2020; 24:100434

3 Thachil J, Lisman T. Pulmonary megakaryocytes in coronavirus disease 2019 (COVID-19): roles in thrombi and fibrosis. Semin Thromb Hemost 2020;46(07):831-834

4 Rodeghiero F. Is ITP a thrombophilic disorder? Am J Hematol 2016;91(01):39-45

5 Vrbensky JR, Nazy I, Toltl LJ, et al. Megakaryocyte apoptosis in immune thrombocytopenia. Platelets 2018;29(07):729-732

6 Hogan M, Geyer JT, Bussel JB. Response to thrombopoietic agents is related to on-treatment bone marrow megakaryocyte morphology in patients with chronic immune thrombocytopenia. Am J Hematol 2019;94(07):E196-E198

7 Kaufman RM, Airo R, Pollack S, Crosby WH. Circulating megakaryocytes and platelet release in the lung. Blood 1965;26(06): 720-731

8 Sharma GK, Talbot IC. Pulmonary megakaryocytes: "missing link" between cardiovascular and respiratory disease? J Clin Pathol 1986;39(09):969-976

9 Lefrançais E, Ortiz-Muñoz G, Caudrillier A, et al. The lung is a site of platelet biogenesis and a reservoir for haematopoietic progenitors. Nature 2017;544(7648):105-109

10 Woods MJ, Greaves M, Lawford PV, Trowbridge EA. Isolation of megakaryocytes from human placentae. Platelets 1994;5(02): 109-112

11 Hansen M, Pedersen NT. Circulating megakaryocytes in patients with pulmonary inflammation and in patients subjected to cholecystectomy. Scand J Haematol 1979;23(03): 211-216

12 Rowley JW, Schwertz H, Weyrich AS. Platelet mRNA: the meaning behind the message. Curr Opin Hematol 2012;19 (05):385-391

13 Roncati L, Ligabue G, Nasillo V, et al. A proof of evidence supporting abnormal immunothrombosis in severe COVID19: naked megakaryocyte nuclei increase in the bone marrow and lungs of critically ill patients. Platelets 2020;31(08): 1085-1089

14 Carsana L, Sonzogni A, Nasr A, Rossi RS, Pellegrinelli A, Zerbi P, et al. Pulmonary post-mortem findings in a series of COVID-19 cases from northern Italy: a two-centre descriptive study. Lancet Infect Dis 2020;20(10):P1135-P1140

15 Wells S, Sissons M, Hasleton PS. Quantitation of pulmonary megakaryocytes and fibrin thrombi in patients dying from burns. Histopathology 1984;8(03):517-527

16 Noetzli LJ, French SL, Machlus KR. New insights into the differentiation of megakaryocytes from hematopoietic progenitors. Arterioscler Thromb Vasc Biol 2019;39(07):1288-1300

17 Kupinski JM, Miller JL. Multimeric analysis of von Willebrand factor in megakaryocytes. Thromb Res 1985;38(06):603-610

18 Goshua G, Pine AB, Meizlish ML, et al. Endotheliopathy in COVID-19-associated coagulopathy: evidence from a singlecentre, cross-sectional study. Lancet Haematol 2020;7(08): e575-e582

19 Taus F, Salvagno G, Canè $S$, et al. Platelets promote thromboinflammation in SARS-CoV-2 pneumonia. Arterioscler Thromb Vasc Biol 2020;40(12):2975-2989

20 Kim WH, Park JB, Jung CW, Kim GS. Rebalanced hemostasis in patients with idiopathic thrombocytopenic purpura. Platelets 2015;26(01):38-42 
21 Casonato A, Fabris F, Boscaro M, Girolami A. Increased factor VIII/vWf levels in patients with reduced platelet number. Blut 1987;54(05):281-288

22 Godfrey CL, Terrinoni I, Laffan M, Crawley J, Cooper N. Elevated plasma Von Willebrand factor and decreased ADAMTS13 antigen levels in patients with immune thrombocytopenia (ITP). Blood 2012;120(21):1096

23 Martinelli N, Montagnana M, Pizzolo F, et al. A relative ADAMTS13 deficiency supports the presence of a secondary microangiopathy in COVID 19. Thromb Res 2020;193:170-172 